\title{
It Started With a Kiss: Monitoring Organelle Interactions and Identifying Membrane Contact Site Components in Plants
}

\author{
Alice L. Baillie ${ }^{1 *}$, Anna-Lena Falz², Stefanie J. Müller-Schüssele ${ }^{2}$ and Imogen Sparkes ${ }^{1}$ \\ ${ }^{1}$ School of Biological Sciences, University of Bristol, Bristol, United Kingdom, ${ }^{2}$ Institut für Nutzpflanzenforschung und \\ Ressourcenschutz (INRES), Rheinische Friedrich-Wilhelms-Universität Bonn, Bonn, Germany
}

\section{OPEN ACCESS}

Edited by:

Pengwei Wang,

Huazhong Agricultural University,

China

Reviewed by:

Abel Rosado,

The University of British Columbia,

Canada

Xiaojuan $\mathrm{Li}$,

Beijing Forestry University, China

*Correspondence:

Alice L. Baillie

Alice.baillie@bristol.ac.uk

Specialty section:

This article was submitted to

Plant Cell Biology,

a section of the journal

Frontiers in Plant Science

Received: 10 February 2020

Accepted: 06 April 2020

Published: 06 May 2020

Citation:

Baillie AL, Falz A-L,

Müller-Schüssele SJ and Sparkes I

(2020) It Started With a Kiss:

Monitoring Organelle Interactions and Identifying Membrane Contact

Site Components in Plants.

Front. Plant Sci. 11:517.

doi: 10.3389/fpls.2020.00517
Organelle movement and interaction are dynamic processes. Interpreting the functional role and mechanistic detail of interactions at membrane contact sites requires careful quantification of parameters such as duration, frequency, proximity, and surface area of contact, and identification of molecular components. We provide an overview of current methods used to quantify organelle interactions in plants and other organisms and propose novel applications of existing technologies to tackle this emerging topic in plant cell biology.

Keywords: membrane contact sites, organelle interactions, Förster resonance energy transfer, optical tweezers, tethers

\section{INTRODUCTION}

Membrane contact sites (MCS) are regions at which transient, physical interactions between organelles occur. These interactions allow exchange of molecules such as signals (e.g., calcium, Csordás et al., 2010) and membrane lipids (Michaud and Jouhet, 2019), and are important for regulating the number and positioning of some organelle types (Phillips and Voeltz, 2016; MuellerSchuessele and Michaud, 2018; White et al., 2020). Direct molecular exchanges between organelles may alternatively be carried out by vesicle-mediated delivery (Scorrano et al., 2019) or by transient interaction between organelles of the same type (e.g., "kiss and run" in mitochondria, Liu et al., 2009). These processes involve the fusion of membranes and are therefore distinct from transient tethering at MCS, which by definition does not involve membrane fusion.

In recent years rapid progress has been made in understanding yeast and mammalian MCS, but studies in plants are less advanced. Some MCS roles will be unique to plants due to the existence of plant-specific compartments, metabolic pathways and processes such as plastids, photorespiration, light/dark adaption and stress responses (Oikawa et al., 2015; Pérez-Sancho et al., 2016).

Here we consider how to define, detect and quantify organelle interactions. We review established techniques for characterizing plant MCS and their protein components, as well as methods that have only been used in non-plant systems to date. For a review of the role of lipids in interactions at MCS, see Petit et al. (2019).

\section{DEFINING AND DETECTING MEMBRANE CONTACT SITES}

Identification of MCS requires that we define them in a measurable way. Multiple, functionally distinct types of contact site can form between given organelle pairs (Siao et al., 2016), with unique 
lipid/protein composition, and characteristic spatial and dynamic properties. Quantifying differences in contact site morphology and dynamics under different environmental conditions can provide clues to MCS function. Direct evidence for a functional, physical interaction requires demonstration of altered molecular exchange between the organelle pair in response to perturbation of MCS formation.

\section{Characterizing Contacts}

It is widely reported that interacting organelles reside within 10-30 nm of one another (Figure 1A), although tethering over distances up to $300 \mathrm{~nm}$ has been reported between mitochondria and the plasma membrane in yeast cells (Klecker et al., 2013; Scorrano et al., 2019). Organelle proximity at MCS will depend on the size and arrangement of tethering proteins. Organelles may also be brought close together through random collisions that are not indicative of interaction (Feng and Kornmann, 2018), especially in vacuolated plant cells in which the cytoplasmic void volume is small relative to the size of the cell. Since the proximity of juxtaposed organelles at MCS is generally much smaller than the maximum resolution of conventional light microscopes ( 200-250 nm), it is impossible to accurately measure the distance between organelles using this technique or to determine whether MCS formation could have occurred. Homotypic interactions present the additional challenge of distinguishing transient membrane contacts from fission or fusion events.

Contact surface area varies depending on the MCS in question (Figure 1B) and can change during development (McFarlane et al., 2017), or in response to biotic (Caplan et al., 2015) or abiotic stresses (Jaipargas et al., 2016; Lee et al., 2019). Changes in total MCS area can be mediated by changes in MCS abundance and/or size. For instance, abundance of a specific type of Endoplasmic reticulum-plasma membrane (ER-PM) contact site in Arabidopsis changed during cell maturation, whereas the average size of parallel membrane stretches (distance $<15 \mathrm{~nm}$ ) quantified via TEM remained largely unchanged at c. $160 \mathrm{~nm}$ (McFarlane et al., 2017). In quantitative TEM analyses, inter-organellar distance thresholds are often used to define MCS and quantify contact area between membranes (Naon et al., 2016; McFarlane et al., 2017). While this may yield a reasonable approximation for MCS number/size, proximity of two membranes is not direct evidence for a functional MCS.

The duration and frequency of MCS formation events (Figure 1C) have been less well studied than their spatial characteristics, probably due to the greater technical challenges of quantifying these parameters. Brief and/or infrequent interactions may be hard to capture, while longlasting interactions may be difficult to monitor for their duration without sample drift and/or fluorescence bleaching problems. Hypothetically, random organelle collisions might be distinguished from regulated membrane interactions by measuring interaction duration, though brief juxtaposition does not necessarily preclude interaction. The dynamics of a given organelle interaction are likely to depend on the functional role of the contact. Varying physiological conditions will alter the demand for exchange of different molecule types, potentially affecting membrane contact frequency and or duration (Helle et al., 2013).

\section{Functional Criteria: Tethering and Molecular Exchange}

While measurements of the parameters above (proximity, surface area, and duration/frequency) cannot provide proof that a functional MCS has formed, each may provide a reasonable basis for further investigation. Evidence of physical tethering and/or molecular exchange is necessary to confirm an interaction. When organelles interact, physical tethers form between them, increasing the force required for their separation (Figure 1D). This is challenging to measure but, techniques such as optical tweezers and shock waves generated from a focused femtosecond laser (see below) can allow demonstration of an increased separation force, which provides biophysical evidence for physical membrane contact (Sparkes, 2016, 2018; Oikawa et al., 2015). Direct demonstration of molecular exchange is another way to confirm a functional organelle interaction (Jouhet et al., 2004; Mehrshahi et al., 2013). Identification of proteins specific to the contact site, such as tethering proteins, functional proteins (e.g., channel proteins) and associated regulatory proteins, can shed light on MCS function and aid visualization through tagging of these MCS components with fluorescent proteins (FPs) (McFarlane et al., 2017). However, many MCS involve multiple tethering proteins, which can prevent mutation of an individual tether from having a measurable phenotypic effect (Scorrano et al., 2019).

\section{TECHNIQUES USED TO STUDY ORGANELLE INTERACTIONS}

\section{Imaging Organelle Dynamics and MCS}

While close organelle proximity is insufficient grounds to confirm an interaction, characterizing juxtaposition duration/frequency (Oikawa et al., 2015; Gao et al., 2016), correlation of movement (Sinclair et al., 2009; Barton et al., 2013; Higa et al., 2014) and contact area (Lee et al., 2019) remains useful, especially when investigating the effects of different environmental treatments (Jaipargas et al., 2016) or manipulating putative tether expression (Mueller and Reski, 2015). Confocal microscopy has become the primary tool of choice, with the wide palate of available fluorophores allowing simultaneous visualization of multiple organelles and proteins (Valm et al., 2017). Electron microscopy (EM) can offer higher resolution, and FPs can be detected using immunogold (Pérez-Sancho et al., 2015; Cui et al., 2016; Wang et al., 2016) or Correlative light and electron microscopy (CLEM), although these approaches are low-throughput. While EM requires sample fixation and therefore abolishes system dynamics, it allows much more accurate quantification of organelle proximity (Caplan et al., 2015) and MCS area, especially if tomography approaches are used to allow $3 \mathrm{D}$ reconstruction (Wang et al., 2019). 


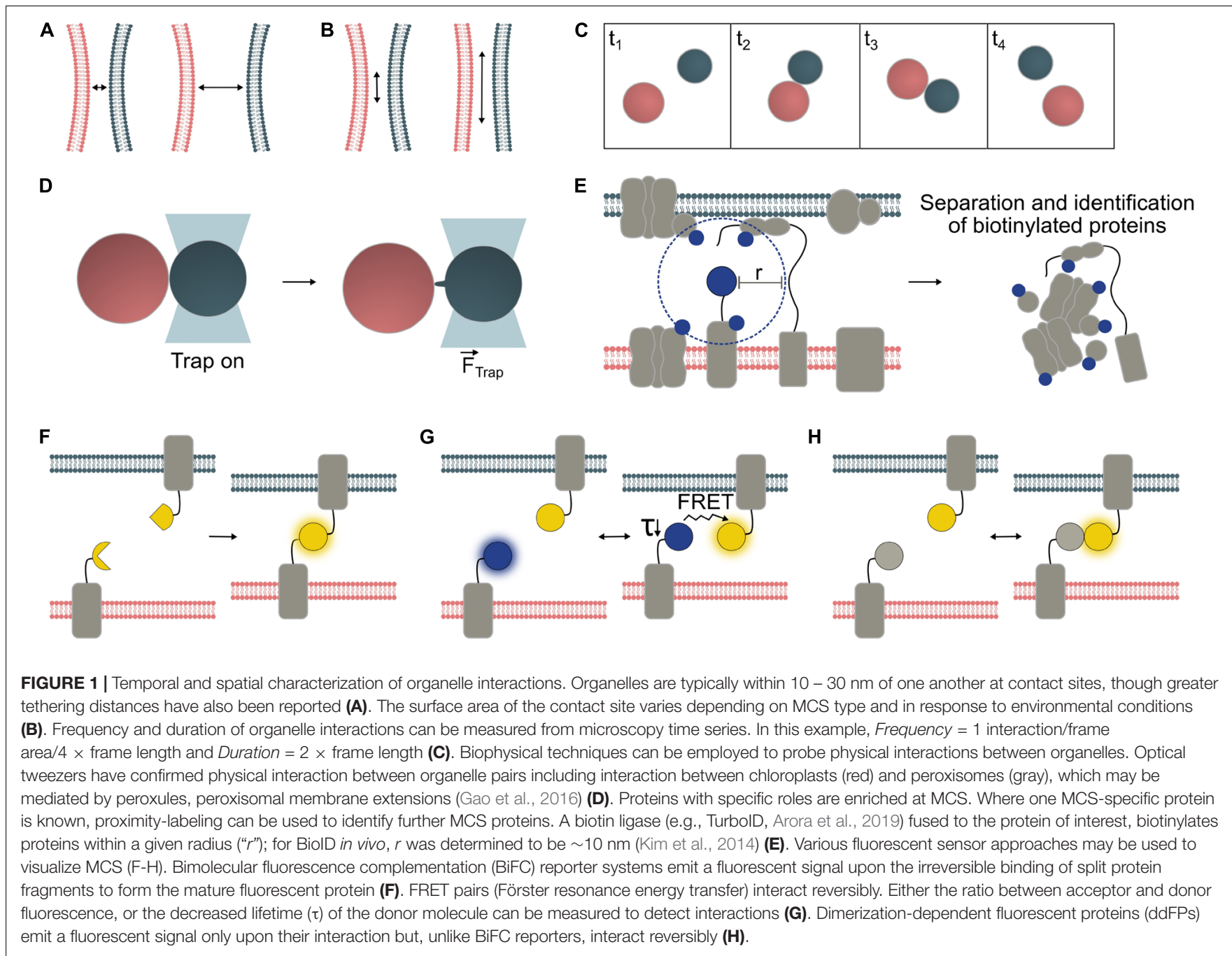

Where MCS tether proteins are known (see below), FP fusions can be created to specifically label MCS, or antibodies raised against tethers for immunogold EM. Lee et al. (2019) tagged known Arabidopsis ER-PM tether Syt1 (Pérez-Sancho et al., 2015) with GFP and compared the localization of this signal with that of MAPPER-GFP, a more ubiquitous ER-PM contact marker adapted from mammalian systems. This demonstrated that the subset of ER-PM contact sites containing SYT1 were involved in increasing ER-PM contact in response to ionic stress.

Super-resolution microscopy techniques use various innovative approaches to overcome the diffraction barrier, allowing imaging of biological structures in greater detail than by conventional light microscopy. Use of these techniques to study MCS components in plants has so far been very limited, though SIM (Structured illumination microscopy) has been applied to image desmotubules of ER in primary plasmodesmata (Knox et al., 2015). Research in non-plant systems is demonstrating further potential for super-resolution monitoring of specific MCS proteins. For example, SIM and dSTORM (direct Stochastic Optical Reconstruction Microscopy) were used to visualize clusters of $\mathrm{MIRO} 1$ and $\mathrm{MIRO} 2$ proteins corresponding to ER-mitochondrial contact sites in mammalian cells (Modi et al., 2019), and ER-PM contact sites were recently investigated using multicolor three-dimensional salvaged fluorescence imaging (Zhang et al., 2020).

\section{Identifying Novel MCS Components}

MCS can be isolated for analysis of lipid and protein content and activity. During cell fractionation and organelle isolation, the presence of MCS leads to the co-purification of membrane regions of interacting organelles, termed "associated membranes." Andersson et al. (2007) isolated chloroplast-associated fragments of ER membrane from pea protoplasts by fractionation. The lipid composition and polypeptide profiles of these plastid-associated membranes (PLAMS) was distinct from the rest of the chloroplast envelope.

Meta-analyses of organelle proteome datasets could power further discovery of novel MCS components. As MCS can copurify in organelle isolations as associated membranes, putative candidates might be found among the contaminants identified in organellar proteomes determined by mass spectrometry (Mueller et al., 2017). Moreover, novel proteomics techniques such as the generation of complexomics datasets for specific 
organelles (Senkler et al., 2017) will allow targeted screening for novel components of specific protein complexes.

Where one contact site component is known, this can be used as the basis for a pull-down assay to identify further proteins within that MCS. Kriechbaumer et al. (2015) performed a GFPtrap assay followed by proteomics using two potential ER-PM contact site proteins (plasmodesmata-localized RTNLB3 and 6) as their bait, thereby identifying 42 and 57 likely interactors for each protein, respectively.

Proximity-labeling has been developed to identify interactors of a protein of interest by biotinylation of its neighbors, separation of these biotin-tagged proteins, and proteomic identification (Figure 1E). APEX2 and BioID are two available biotinylation probes, and both have been used to discover new MCS protein components in mammalian cells (Jing et al., 2015; Lam et al., 2015; van Vliet et al., 2017). BioID has also been used in plants, though not specifically with MCS proteins (Khan et al., 2018). Split variants of both APEX2 (Han et al., 2019) and BioID (De Munter et al., 2017) allow greater specificity through interaction-dependent proximitylabeling, and the former has been applied to ER-PM contact sites in mammalian cells. In plants, established BioID probes show low activity since their optimal working temperature is higher than the plant growth temperature. However, a recently described promiscuous mutant of biotin ligase, TurboID, is less affected by low temperatures compared to earlier BioID variants and has be shown to work efficiently in planta (Branon et al., 2018; Arora et al., 2019). Furthermore, a newly reported split-TurboID probe allowed contact-dependent proximity-labelling at ERmitochondria contact sites in mammalian cells (Cho et al., 2020). Therefore this system offers promise for proximity-labeling in plant MCS as well.

\section{Detecting and Monitoring MCS With Fluorescent Probes}

Studying the interactions of specific protein pairs is possible through FRET (Förster resonance energy transfer) or split fluorescent reporter systems such as BiFC (Bimolecular fluorescence complementation) (Kerppola, 2006; Xing et al., 2016). Both techniques translate proximity into a fluorescent signal, so have potential for visualization of MCS between two organelles. However, fusion partners to detect MCS must be carefully chosen to ensure compatibility with the chosen FP system, specific targeting to the outer membrane of the organelle of interest, and cytosolic orientation of the FP tags.

In BiFC (Figure 1F), a fluorescent signal is emitted if fragments of a fluorescent protein come close enough to allow reassembly and chromophore formation (Magliery et al., 2005). Split fluorescent reporter systems have been successfully applied for MCS detection in animal and fungal cells (Cieri et al., 2018; Shai et al., 2018; Yang et al., 2018) but have been little used in plant systems to date. While they are sensitive tools, spontaneous assembly of BiFC probes can cause false-positive artifacts and non-specific signals. Hence, selection of appropriate negative controls and/or use of ratiometric systems is essential (Grefen and Blatt, 2012; Xing et al., 2016), and protein interactions should be verified by another, independent technique. In most split fluorescent reporter systems, such as split-GFP and YFP-derived split-Venus (Figure 1F), complementation of the fluorophore fragments is irreversible (Magliery et al., 2005), so the duration of the interaction and subsequent dynamics cannot be quantified. This property can, however, be used to deliberately generate "artificial tethers" to manipulate MCS by fixation or expansion. Tao et al. (2019a,b). reported the creation of artificial ERPM and PM-multivesicular body/tonoplast tethers using splitVenus in plants.

In contrast, FRET (Figure 1G) between a suitable FP pair is fully reversible. Its efficiency depends on the distance between the fluorophores and the specific Förster radius of the FRET pair, covering detection ranges of $\sim 3-10 \mathrm{~nm}$ (for review, see Algar et al., 2019). FRET can be detected by monitoring the acceptor/donor fluorescence intensity ratio, or the decrease in fluorescence lifetime of the donor by Fluorescence Lifetime Imaging (FLIM) (for review, see Xing et al., 2016). Due to its reversibility, FRET can be measured in dynamic systems, though the required imaging time for FLIM (several seconds per image) may limit detection of brief contact events using this approach. One mammalian cell study using rapamycin-inducible tethers, monitored changes in ER-mitochondrial contact site abundance in response to inducer application at high spatial resolution at the organelle level by measuring FRET ratios (Csordás et al., 2010).

FRET-FLIM has been used in plant cells to demonstrate interactions between specific protein partners at MCS in Golgi stacks (Osterrieder et al., 2009). Cells were treated with latrunculin B to depolymerize actin and stop Golgi movement. Some of these interactions occurred only in a subset of Golgi stacks, demonstrating that an in planta approach adds additional, spatial information compared to in vitro, biochemical studies. In mammalian cells, Venditti et al. (2019) used a FLIM approach to identify ER-Golgi tethers by tagging fluorophores to homogeneously distributed proteins in the membranes of these organelles and systematically depleting tethering candidates by siRNA (small interfering RNA). While the regions of interest on which they conducted their FLIM measurements were not resolved to the level of MCS or even individual Golgi bodies, they paired these measurements with TEM images to examine in detail changes in MCS structures associated with various cell treatments.

Dimerization-dependent FPs (ddFPs; Figure 1H) offer another way to reversibly detect proximity, though they produce low signal levels. While unsuitable for dynamically studying interactions between freely diffusing proteins, ddFPs can be applied to monitor interactions between membrane-associated proteins, as demonstrated with ER-mitochondrial MCS in human and mouse cells (Alford et al., 2012).

FRET, BiFC and ddFPs all depend on the ability of the interaction partners to reach one another, so selecting fusion partners and designing constructs may be problematic if the diameter of the endogenous MCS tethering complex is unknown. Appropriate choice of linker length between the fluorescent tags and proteins of interest is key for successful FRET and split fluorescence assays for protein-protein interactions. Cieri et al. (2018) made two versions of their 
TABLE 1 | Outcomes of current technologies, summarizing our knowledge to date of organelle interactions and their molecular components in plants.

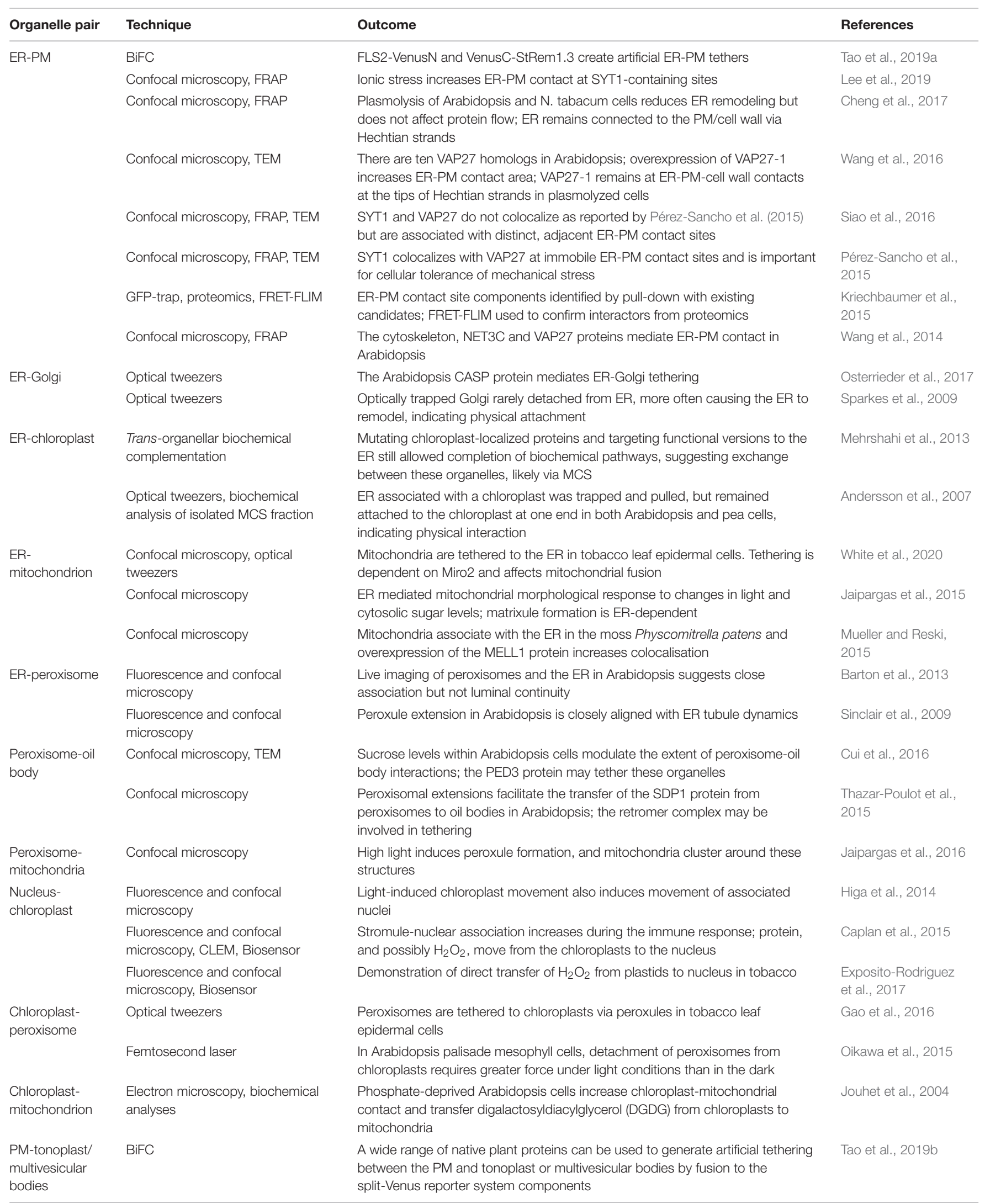


self-assembling GFP-based probe to detect narrow and wide ERmitochondrial contact sites, while Várnai et al. (2007) turned this challenge into a tool, using constructs that varied in their linker length to "measure" ER-PM contact distance in mammalian cells.

\section{Measuring Organelle Tethering Forces}

Biophysical methods have been applied to determine physical association between plant organelles. Oikawa et al. (2015) used a femtosecond laser to generate shock waves to quantify the physical interaction between chloroplasts and peroxisomes. This same organelle pairing has been analyzed using optical tweezers (Gao et al., 2016), as have ER-Golgi (Sparkes et al., 2009), ER-chloroplast (Andersson et al., 2007) and ERmitochondria interactions (White et al., 2020). Optical tweezers provide submicron accuracy to trap and "target" a single organelle and micro-manipulate its position relative to other structures (Figure 1D). In this way, the ER-Golgi tethering function of the Arabidopsis CASP protein was discovered (Osterrieder et al., 2017).

\section{Demonstrating Molecular Exchange at MCS}

To understand the functions of MCS we must identify the processes taking place during organelle contact (Helle et al., 2013). Care must be taken to distinguish MCS-mediated molecular exchange from exchange via alternative mechanisms such as vesicle trafficking (Jouhet et al., 2004, 2019). In the case of protein movement from one organelle to another, fluorescent tags and confocal imaging can demonstrate exchange, though not elucidate its mechanism (Caplan et al., 2015; Thazar-Poulot et al., 2015). Demonstrating exchange of nonprotein molecules can present additional challenges as they cannot be directly fluorescently tagged. Genetically encoded ROS biosensors have been used for parallel monitoring of $\mathrm{H}_{2} \mathrm{O}_{2}$ levels in plastids and juxtaposed nuclei in tobacco epidermal cells (Caplan et al., 2015; Exposito-Rodriguez et al., 2017). Jouhet et al. (2004) showed transfer of the glycolipid digalactosyldiacylglycerol (DGDG) from its site of synthesis in plastids to mitochondria in phosphate-deprived plants using biochemical approaches and immunolabelling with EM over time series. Mehrshahi et al. (2013) successfully employed a transorganellar complementation approach, suggesting biochemical continuity between ER and plastids by mutating enzymes in one organelle, targeting functional versions of each enzyme to the other, and showing that the product of the metabolic pathway was still produced.

\section{REFERENCES}

Alford, S. C., Ding, Y., Simmen, T., and Campbell, R. E. (2012). Dimerizationdependent green and yellow fluorescent proteins. ACS Synth. Biol. 1, 569-575. doi: $10.1021 /$ sb300050j

Algar, W. R., Hildebrandt, N., Vogel, S. S., and Medintz, I. L. (2019). FRET as a biomolecular research tool - understanding its potential while avoiding pitfalls. Nat. Methods 16, 815-829. doi: 10.1038/s41592-019-0530-8

Andersson, M. X., Goksör, M., and Sandelius, A. S. (2007). Optical manipulation reveals strong attracting forces at membrane contact sites between endoplasmic

\section{OUTLOOK}

Novel microscopy and molecular biology technologies will continue to expand our toolbox for MCS studies. Tracking and localization microscopy (TALM) and Single particle tracking (SPT) techniques, which provide nano-scale information on the localization and movement trajectories of individual FP fusions, have been used to monitor mitochondrial proteins within membrane microdomains in mammalian cells (Appelhans and Busch, 2017) and could similarly be used to monitor functional MCS proteins and/or tethers. Development of reversible split fluorescent probes, such as the recently reported splitFAST (Fluorescence-activating and absorption-shifting tag) system (Tebo and Gautier, 2019), will overcome the challenge of visualizing transient protein interactions in real time. Direct quantification of tethering forces may also become possible through application of genetically encoded, FRET-based force sensors (Freikamp et al., 2016).

Much remains to be learned about the function and composition of MCS, especially in plant systems. Relatively few molecular components of plant MCS have been identified and evidence of the molecular fluxes that they facilitate is similarly sparse (Table 1). Some examples of MCS responses to environmental changes have been recorded, but the molecular details of these responses and their functional significance remain elusive. We anticipate continued rapid progress in this exciting field driven by innovation in microscopic and molecular technologies.

\section{AUTHOR CONTRIBUTIONS}

All authors contributed toward the conception and writing of the manuscript. A-LF generated the figures.

\section{FUNDING}

$\mathrm{AB}$ and IS are grateful for support from the Leverhulme Trust (RPG-2015-106), STFC (PM-1216) and Bristol Centre for Agricultural Innovation. A-LF and SM-S are grateful for support by the Deutsche Forschungsgemeinschaft (DFG, MU 4137/1-1) as part of the package PAK918 ("Plant Mitochondria in New Light"), and via the research training group RTG2064 ("Water use efficiency and drought stress responses: From Arabidopsis to barley").

reticulum and chloroplasts. J. Biol. Chem. 282, 1170-1174. doi: 10.1074/jbc. M608124200

Appelhans, T., and Busch, K. B. (2017). Dynamic imaging of mitochondrial membrane proteins in specific sub-organelle membrane locations. Biophys. Rev. 9, 345-352. doi: 10.1007/s12551-017-0287-1

Arora, D. B., Abel, N., Liu, C., Van Damme, P., Dai Vu, L., Tornkvist, A., et al. (2019). Establishment of proximity-dependent biotinylation approaches in different plant model systems. bioRxiv [Preprint], doi: 10.1101/701425

Barton, K., Mathur, N., and Mathur, J. (2013). Simultaneous live-imaging of peroxisomes and the ER in plant cells suggests contiguity but no luminal 
continuity between the two organelles. Front. Physiol. 4:196. doi: 10.3389/fphys. 2013.00196

Branon, T. C., Bosch, J. A., Sanchez, A. D., Udeshi, N. D., Svinkina, T., Carr, S. A., et al. (2018). Efficient proximity labeling in living cells and organisms with TurboID. Nat. Biotechnol. 36, 880-887. doi: 10.1038/nbt.4201

Caplan, J. L., Kumar, A. S., Park, E., Padmanabhan, M. S., Hoban, K., Modla, S., et al. (2015). Chloroplast stromules function during innate immunity. Dev. Cell 34, 45-57. doi: 10.1016/j.devcel.2015.05.011

Cheng, X., Lang, I., Adeniji, O. S., and Griffing, L. (2017). Plasmolysisdeplasmolysis causes changes in endoplasmic reticulum form, movement, flow, and cytoskeletal association. J. Exp. Bot. 68, 4075-4087. doi: 10.1093/jxb/erx243

Cho, K. F., Branon, T. C., Rajeev, S., Svinkina, T., Udeshi, N. D., Thoudam, T., et al. (2020). Split-TurboID enables contact-dependent proximity labeling in cells. bioRxiv [Preprint], doi: 10.1101/2020.03.11.988022

Cieri, D., Vicario, M., Giacomello, M., Vallese, F., Filadi, R., Wagner, T., et al. (2018). SPLICS: a split green fluorescent protein-based contact site sensor for narrow and wide heterotypic organelle juxtaposition. Cell Death Differ. 25, 1131-1145. doi: 10.1038/s41418-017-0033-Z

Csordás, G., Várnai, P., Golenár, T., Roy, S., Purkins, G., Schneider, T. G., et al. (2010). Imaging interorganelle contacts and local calcium dynamics at the ermitochondrial interface. Mol. Cell 39, 121-132. doi: 10.1016/j.molcel.2010.06. 029

Cui, S., Hayashi, Y., Otomo, M., Mano, S., Oikawa, K., Hayashi, M., et al. (2016). Sucrose production mediated by lipid metabolism suppresses the physical interaction of peroxisomes and oil bodies during germination of Arabidopsis thaliana. J. Biol. Chem. 291, 19734-19745. doi: 10.1074/jbc.M116.748814

De Munter, S., Görnemann, J., Derua, R., Lesage, B., Qian, J., Heroes, E., et al. (2017). Split-BioID: a proximity biotinylation assay for dimerization-dependent protein interactions. FEBS Lett. 591, 415-424. doi: 10.1002/1873-3468.12548

Exposito-Rodriguez, M., Laissue, P. P., Yvon-Durocher, G., Smirnoff, N., and Mullineaux, P. M. (2017). Photosynthesis-dependent $\mathrm{H} 2 \mathrm{O} 2$ transfer from chloroplasts to nuclei provides a high-light signalling mechanism. Nat. Commun. 8:49. doi: 10.1038/s41467-017-00074-w

Feng, Q., and Kornmann, B. (2018). Mechanical forces on cellular organelles. J. Cell Sci. 131:218479. doi: 10.1242/jcs.218479

Freikamp, A., Cost, A. L., and Grashoff, C. (2016). The piconewton force awakens: quantifying mechanics in cells. Trends Cell Biol. 26, 838-847. doi: 10.1016/j.tcb. 2016.07.005

Gao, H., Metz, J., Teanby, N. A., Ward, A. D., Botchway, S. W., Coles, B., et al. (2016). In vivo quantification of peroxisome tethering to chloroplasts in tobacco epidermal cells using optical tweezers. Plant Physiol. 170, 263-272. doi: 10.1104/ pp.15.01529

Grefen, C., and Blatt, M. R. (2012). A 2in1 cloning system enables ratiometric bimolecular fluorescence complementation (rBiFC). Biotechniques 53, 311-314. doi: $10.2144 / 000113941$

Han, Y., Branon, T. C., Martell, J. D., Boassa, D., Shechner, D., Ellisman, M. H., et al. (2019). Directed evolution of split APEX2 peroxidase. ACS Chem. Biol. 14, 619-635. doi: 10.1021/acschembio.8b00919

Helle, S. C. J., Kanfer, G., Kolar, K., Lang, A., Michel, A. H., and Kornmann, B. (2013). Organization and function of membrane contact sites. Biochim. Biophys. Acta Mol. Cell Res. 1833, 2526-2541. doi: 10.1016/j.bbamcr.2013.01.028

Higa, T., Suetsugu, N., Kong, S. G., and Wada, M. (2014). Actin-dependent plastid movement is required for motive force generation in directional nuclear movement in plants. Proc. Natl. Acad. Sci. U.S.A. 111, 4327-4331. doi: 10.1073/ pnas. 1317902111

Jaipargas, E. A., Barton, K. A., Mathur, N., and Mathur, J. (2015). Mitochondrial pleomorphy in plant cells is driven by contiguous ER dynamics. Front. Plant Sci. 6:783. doi: 10.3389/fpls.2015.00783

Jaipargas, E. A., Mathur, N., Daher, F. B., Wasteneys, G. O., and Mathur, J. (2016). High light intensity leads to increased peroxule-mitochondria interactions in plants. Front. Cell Dev. Biol. 4:6. doi: 10.3389/fcell.2016.00006

Jing, J., He, L., Sun, A., Quintana, A., Ding, Y., Ma, G., et al. (2015). Proteomic mapping of ER-PM junctions identifies STIMULATW as a regulator of Cs2+ influx. Nat. Cell Biol. 17, 1339-1347. doi: 10.1038/ncb3234.Proteomic

Jouhet, J., Gros, V., and Michaud, M. (2019). "Measurement of lipid transport in mitochondria by the MTL complex," in Intracellular Lipid Transport: Methods and Protocols, ed. G. Drin (New York, NY: Springer), 69-93. doi: 10.1007/9781-4939-9136-5_7
Jouhet, J., Maréchal, E., Baldan, B., Bligny, R., Joyard, J., and Block, M. A. (2004). Phosphate deprivation induces transfer of DGDG galactolipid from chloroplast to mitochondria. J. Cell Biol. 167, 863-874. doi: 10.1083/jcb.200407022

Kerppola, T. K. (2006). Design and implementation of bimolecular fluorescence complementation $(\mathrm{BiFC})$ assays for the visualization of protein interactions in living cells. Nat. Protoc. 1, 1278-1286. doi: 10.1038/nprot.2006.201

Khan, M., Youn, J. Y., Gingras, A. C., Subramaniam, R., and Desveaux, D. (2018). In planta proximity dependent biotin identification (BioID). Sci. Rep. 8, 2-9. doi: 10.1038/s41598-018-27500-3

Kim, D. I., Birendra, K. C., Zhu, W., Motamedchaboki, K., Doye, V., and Roux, K. J. (2014). Probing nuclear pore complex architecture with proximity-dependent biotinylation. Proc. Natl. Acad. Sci. U.S.A. 111, 2453-2461. doi: 10.1073/pnas. 1406459111

Klecker, T., Scholz, D., Förtsch, J., and Westermann, B. (2013). The yeast cell cortical protein Numl integrates mitochondrial dynamics into cellular architecture. J. Cell Sci. 126, 2924-2930. doi: 10.1242/jcs.126045

Knox, K., Wang, P., Kriechbaumer, V., Tilsner, J., Frigerio, L., Sparkes, I., et al. (2015). Putting the squeeze on plasmodesmata: a role for reticulons in primary plasmodesmata formation. Plant Physiol. 168, 1563-1572. doi: 10.1104/pp.15. 00668

Kriechbaumer, V., Botchway, S. W., Slade, S. E., Knox, K., Frigerio, L., Oparka, K., et al. (2015). Reticulomics: protein-protein interaction studies with two plasmodesmata-localized reticulon family proteins identify binding partners enriched at plasmodesmata, endoplasmic reticulum, and the plasma membrane. Plant Physiol. 169, 1933-1945. doi: 10.1104/pp.15.01153

Lam, S. S., Martell, J. D., Kamer, K. J., Deerinck, T. J., Ellisman, M. H., Mootha, V. K., et al. (2015). Directed evolution of APEX2 for electron microscopy and proteomics. Nat. Methods 12, 51-54. doi: 10.4172/2157-7633.1000305

Lee, E., Vanneste, S., Pérez-Sancho, J., Benitez-Fuente, F., Strelau, M., Macho, A. P., et al. (2019). Ionic stress enhances ER-PM connectivity via phosphoinositideassociated SYT1 contact site expansion in Arabidopsis. Proc. Natl. Acad. Sci. U.S.A. 116, 1420-1429. doi: 10.1073/pnas. 1818099116

Liu, X., Weaver, D., Shirihai, O., and Hajnóczky, G. (2009). Mitochondrial kiss-and-run: interplay between mitochondrial motility and fusion-fission dynamics. EMBO J. 28, 3074-3089. doi: 10.1038/emboj.2009.255

Magliery, T. J., Wilson, C. G. M., Pan, W., Mishler, D., Ghosh, I., Hamilton, A. D., et al. (2005). Detecting protein - protein interactions with a green fluorescent protein fragment reassembly trap: scope and mechanism. J Am Chem Soc 127, 146-157. doi: 10.1021/ja046699g

McFarlane, H. E., Lee, E. K., Van Bezouwen, L. S., Ross, B., Rosado, A., and Samuels, A. L. (2017). Multiscale structural analysis of plant ER-PM contact sites. Plant Cell Physiol. 58, 478-484. doi: 10.1093/pcp/pcw224

Mehrshahi, P., Stefano, G., Andaloro, J. M., Brandizzi, F., Froehlich, J. E., and DellaPenna, D. (2013). Transorganellar complementation redefines the biochemical continuity of endoplasmic reticulum and chloroplasts. Proc. Natl. Acad. Sci. U.S.A. 110, 12126-12131. doi: 10.1073/pnas.1306331110

Michaud, M., and Jouhet, J. (2019). Lipid trafficking at membrane contact sites during plant development and stress response. Front. Plant Sci. 10:2. doi: 10. 3389/fpls.2019.00002

Modi, S., López-Doménech, G., Halff, E. F., Covill-Cooke, C., Ivankovic, D., Melandri, D., et al. (2019). Miro clusters regulate ER-mitochondria contact sites and link cristae organization to the mitochondrial transport machinery. Nat. Commun. 10:4399. doi: 10.1038/s41467-019-12382-4

Mueller, S. J., Hoernstein, S. N. W., and Reski, R. (2017). "Approaches to characterize organelle, compartment, or structure purity," in Isolation of Plant Organelles and Structures: Methods and Protocols, eds N. L. Taylor and A. H. Millar (New York, NY: Springer), 13-28. doi: 10.1007/978-1-4939-6533-5_2

Mueller, S. J., and Reski, R. (2015). Mitochondrial dynamics and the ER: the plant perspective. Front. Cell Dev. Biol. 3:78. doi: 10.3389/fcell.2015.00078

Mueller-Schuessele, S. J., and Michaud, M. (2018). Plastid transient and stable interactions with other cell compartments. Methods Mol. Biol. 1829, 87-109.

Naon, D., Zaninello, M., Giacomello, M., Varanita, T., and Grespi, F. (2016). Critical reappraisal confirms that Mitofusin 2 is an endoplasmic reticulum mitochondria tether. Proc. Natl. Acad. Sci. U.S.A. 113, 11249-11254. doi: 10. 1073/pnas.1606786113

Oikawa, K., Matsunaga, S., Mano, S., Kondo, M., Yamada, K., Hayashi, M., et al. (2015). Physical interaction between peroxisomes and chloroplasts elucidated by in situ laser analysis. Nat. Plants 1:15035. doi: 10.1038/nplants.2015.35 
Osterrieder, A., Carvalho, C. M., Latijnhouwers, M., Johansen, J. N., Stubbs, C., Botchway, S., et al. (2009). Fluorescence lifetime imaging of interactions between Golgi tethering factors and small GTPases in plants. Traffic 10, 10341046. doi: 10.1111/j.1600-0854.2009.00930.x

Osterrieder, A., Sparkes, I. A., Botchway, S. W., Ward, A., Ketelaar, T., de Ruijter, N., et al. (2017). Stacks off tracks: a role for the golgin AtCASP in plant endoplasmic reticulum-Golgi apparatus tethering. J. Exp. Bot. Bot. 68, 33393350. doi: 10.1093/jxb/erx167

Pérez-Sancho, J., Tilsner, J., Samuels, A. L., Botella, M. A., Bayer, E. M., and Rosado, A. (2016). Stitching organelles: organization and function of specialized membrane contact sites in plants. Trends Cell Biol. 26, 705-717. doi: 10.1016/j. tcb.2016.05.007

Pérez-Sancho, J., Vanneste, S., Lee, E., McFarlane, H. E., Esteban del Valle, A., Valpuesta, V., et al. (2015). The Arabidopsis synaptotagminl is enriched in endoplasmic reticulum-plasma membrane contact sites and confers cellular resistance to mechanical stresses. Plant Physiol. 168, 132-143. doi: 10.1104/pp. 15.00260

Petit, J. D., Immel, F., Lins, L., and Bayer, E. M. (2019). Lipids or proteins: who is leading the dance at membrane contact sites? Front. Plant Sci. 10:1-10. doi: $10.3389 /$ fpls. 2019.00198

Phillips, M. J., and Voeltz, G. K. (2016). Structure and function of ER membrane contact sites with other organelles. Nat. Rev. Mol. Cell Biol. 17, 69-82. doi: 10.1038/nrm.2015.8

Scorrano, L., De Matteis, M. A., Emr, S., Giordano, F., Hajnóczky, G., Kornmann, B., et al. (2019). Coming together to define membrane contact sites. Nat. Commun. 10:1287. doi: 10.1038/s41467-019-09253-3

Senkler, J., Senkler, M., Eubel, H., Hildebrandt, T., Lengwenus, C., Schertl, P., et al. (2017). The mitochondrial complexome of Arabidopsis thaliana. Plant J. 89, 1079-1092. doi: 10.1111/tpj.13448

Shai, N., Yifrach, E., Van Roermund, C. W. T., Cohen, N., Bibi, C., Ijlst, L., et al. (2018). Systematic mapping of contact sites reveals tethers and a function for the peroxisome-mitochondria contact. Nat. Commun. 9:1761. doi: 10.1038/s41467018-03957-8

Siao, W., Wang, P., Voigt, B., Hussey, P. J., and Baluska, F. (2016). Arabidopsis SYT1 maintains stability of cortical endoplasmic reticulum networks and VAP27-1-enriched endoplasmic reticulum-plasma membrane contact sites. J. Exp. Bot. 67, 6161-6171. doi: 10.1093/jxb/erw381

Sinclair, A. M., Trobacher, C. P., Mathur, N., Greenwood, J. S., and Mathur, J. (2009). Peroxule extension over ER-defined paths constitutes a rapid subcellular response to hydroxyl stress. Plant J. 59, 231-242. doi: 10.1111/j.1365-313X. 2009.03863.x

Sparkes, I. (2016). Using optical tweezers to characterize physical tethers at membrane contact sites: grab it, pull it, set it free? Front. Cell Dev. Biol. 4:22. doi: $10.3389 /$ fcell.2016.00022

Sparkes, I. (2018). Lessons from optical tweezers: quantifying organelle interactions, dynamics and modelling subcellular events. Curr. Opin. Plant Biol. 46, 55-61. doi: 10.1016/j.pbi.2018.07.010

Sparkes, I. A., Ketelaar, T., De Ruijter, N. C. A., and Hawes, C. (2009). Grab a golgi: laser trapping of Golgi bodies reveals in vivo interactions with the endoplasmic reticulum. Traffic 10, 567-571. doi: 10.1111/j.1600-0854.2009.00891.x

Tao, K., Waletich, J. R., Arredondo, F., and Tyler, B. M. (2019a). Manipulating endoplasmic reticulum-plasma membrane tethering in plants through fluorescent protein complementation. Front. Plant Sci. 10:635. doi: 10.3389/fpls. 2019.00635

Tao, K., Waletich, J. R., Wise, H., Arredondo, F., Tyler, B. M., and Tyler, B. M. (2019b). Tethering of multi-vesicular bodies and the Tonoplast to the plasma membrane in plants. Front. Plant Sci. 10:636. doi: 10.3389/fpls.2019.00636
Tebo, A. G., and Gautier, A. (2019). A split fluorescent reporter with rapid and reversible complementation. Nat. Commun. 10:2822. doi: 10.1038/s41467-01910855-0

Thazar-Poulot, N., Miquel, M., Fobis-Loisy, I., and Gaude, T. (2015). Peroxisome extensions deliver the Arabidopsis SDP1 lipase to oil bodies. Proc. Natl. Acad. Sci. U.S.A. 112, 4158-4163. doi: 10.1073/pnas.1403322112

Valm, A. M., Cohen, S., Legant, W. R., Melunis, J., Hershberg, U., Wait, E., et al. (2017). Applying systems-level spectral imaging and analysis to reveal the organelle interactome. Nature 546, 162-167. doi: 10.1038/nature22369

van Vliet, A. R., Giordano, F., Gerlo, S., Segura, I., Van Eygen, S., Molenberghs, G., et al. (2017). The ER stress sensor PERK coordinates ER-plasma membrane contact site formation through interaction with filamin-A and F-Actin remodeling. Mol. Cell 65, 885-899. doi: 10.1016/j.molcel.2017.01.020

Várnai, P., Tóth, B., Tóth, D. J., Hunyady, L., and Balla, T. (2007). Visualization and manipulation of plasma membrane-endoplasmic reticulum contact sites indicates the presence of additional molecular components within the STIM1Orail complex. J. Biol. Chem. 282, 29678-29690. doi: 10.1074/jbc.M704339200

Venditti, R., Rega, L. R., Masone, M. C., Santoro, M., Polishchuk, E., Sarnataro, D., et al. (2019). Molecular determinants of ER-Golgi contacts identified through a new FRET-FLIM system. J. Cell Biol. 218, 1055-1065. doi: 10.1083/jcb. 201812020

Wang, P., Hawkins, T. J., Richardson, C., Cummins, I., Deeks, M. J., Sparkes, I., et al. (2014). The plant cytoskeleton, NET3C, and VAP27 mediate the link between the plasma membrane and endoplasmic reticulum. Curr. Biol. 24, 1397-1405. doi: 10.1016/j.cub.2014.05.003

Wang, P., Liang, Z., and Kang, B. H. (2019). Electron tomography of plant organelles and the outlook for correlative microscopic approaches. New Phytol. 223, 1756-1761. doi: 10.1111/nph.15882

Wang, P., Richardson, C., Hawkins, T. J., Sparkes, I., Hawes, C., and Hussey, P. J. (2016). Plant VAP27 proteins: domain characterization, intracellular localization and role in plant development. New Phytol. 210, 1311-1326. doi: $10.1111 /$ nph.13857

White, R. R., Lin, C., Leaves, I., Castro, I. G., Metz, J., Bateman, B. C., et al. (2020). Miro2 tethers the ER to mitochondria to promote mitochondrial fusion in tobacco leaf epidermal cells. Commun. Biol. 3, 1-8. doi: 10.1038/s42003-0200872-x

Xing, S., Wallmeroth, N., Berendzen, K. W., and Grefen, C. (2016). Techniques for the analysis of protein-protein interactions in vivo. Plant Physiol. 171, 727-758. doi: $10.1104 /$ pp. 16.00470

Yang, Z., Zhao, X., Xu, J., Shang, W., and Tong, C. (2018). A novel fluorescent reporter detects plastic remodeling of mitochondria-ER contact sites. J. Cell Sci. 131:208686. doi: 10.1242/jcs.208686

Zhang, Y., Schroeder, L. K., Lessard, M. D., Kidd, P., Chung, J., Song, Y., et al. (2020). Nanoscale subcellular architecture revealed by multicolor threedimensional salvaged fluorescence imaging. Nat. Methods 17, 225-231. doi: 10.1038/s41592-019-0676-4

Conflict of Interest: The authors declare that the research was conducted in the absence of any commercial or financial relationships that could be construed as a potential conflict of interest.

Copyright (c) 2020 Baillie, Falz, Müller-Schüssele and Sparkes. This is an open-access article distributed under the terms of the Creative Commons Attribution License (CC BY). The use, distribution or reproduction in other forums is permitted, provided the original author(s) and the copyright owner(s) are credited and that the original publication in this journal is cited, in accordance with accepted academic practice. No use, distribution or reproduction is permitted which does not comply with these terms. 